\title{
Prevention of Infection in Lupus Patients
}

\author{
M EL Fane*, M Sodqi, A Chakib and K Marhoum EL Filali \\ Unit of Infectiology Diseases, UHC Ibn Rochd, Casablanca, Morocco
}

"Corresponding author: M EL Fane, University Ibn Zohr, Agadir, Morocco, Tel: 212 660832362; E-mail: elfanemouna@gmail.com

Received date: December 26, 2016; Accepted date: January 19, 2017; Published date: January 25,2017

Copyright: ( 2017 EL Fane M, et al. This is an open-access article distributed under the terms of the Creative Commons Attribution License, which permits unrestricted use, distribution, and reproduction in any medium, provided the original author and source are credited.

\begin{abstract}
Despite the many advances in the treatment of systemic lupus erythematous (SLE), infection remains one of the leading causes of morbidity and mortality of patients. The reasons for the high incidence of infection are immunosuppressive therapy and immune disturbances of lupus itself. Bacterial infections are most frequent, followed by viral and fungal infections. Vaccination is the most important tool in the prevention of infections specially influenza and Streptococcus pneumonia infections. Prophylaxis of tuberculosis and pneumocystosis are also recommended to prevent those deadly infections. In this review, we aim to give an overview of the prevention means of infections in SLE.
\end{abstract}

Keywords: Lupus; Infection; Vaccination; Chemoprophylaxis

\section{Introduction}

Systemic lupus erythematosus (SLE) is an autoimmune disease, characterized by dysregulation of the innate and adaptive immune systems, production of autoantibodies, and tissue damage resulting from chronic inflammation $[1,2]$. The treatment includes the use of non-steroidal anti-inflammatory and anti-malarial drugs, corticosteroids and immunosuppressive agents [3]. Despite great improvements in reducing mortality in SLE, the mortality due to infections remains unchanged, constituting the cause of death in 1 out of 3 SLE patients [3-5]. Multiple risk factors for infection have been reported. They include immunological disorders due to lupus and its therapy, particularly immunosuppressive and cytotoxic medications [5,6]. Lupus disease activity, measured by the SLE disease activity index (SLEDAI), is also an important risk factor for infection [7]. The risk is also dependent on the degree of immunosuppression [8-10]. SLE patients may develop common bacterial opportunistic infections particularly of the respiratory and urinary tracts [3]. Opportunistic infections such as pneumocystis (PCP) are associated with mortality as high as $46 \%[5,6]$. Debilitating viral infections, such as herpes zoster and cytomegalovirus (CMV), are also seen at significantly higher rates in patients with SLE [11]. In this review, we aim to give an overview of the prevention means of infections in SLE.

\section{How to Reduce the Risk of Infectious Complications in Patients with SLE?}

\section{Vaccine recommendations}

Vaccination is the most important tool in the prevention of infections in SLE patients [12]. The European league against rheumatism is recommended that patients with LES receive vaccinations within a period of clinical remission $[13,14]$. Non-live vaccines can be administered independent of medication use. Inactivated live vaccines are best avoided in patients receiving immunosuppressive agents, including glucocorticoids at daily doses over $20 \mathrm{mg}$. Live vaccines are contraindicated in SLE patients with active disease or on high-dose immunosuppressive therapy as they can result in infection [13,15-17]. Many vaccines, including HPV, hepatitis $B$, pneumococcal vaccine, and influenza vaccine, have been shown to be immunogenic in the SLE population [18]. Although these vaccines have found to be generally safe and well tolerated by SLE patients, lupus-like syndrome and other autoimmune phenomena such as induction of lupus anticoagulants, anti-Ro/La and anti-smith antibodies have been reported following various immunizations [16]. Likewise, vaccinations may be less efficacious in SLE patients, as they have reduced primary and secondary immune responses upon antigenic challenge, in part due to immunosuppressive therapy $[17,18]$.

\section{Influenza vaccination}

The prevention of influenza infections with vaccination (trivalentinactivated) should receive particular attention considering the danger coming from the annual possible stimulation of a dysregulated immune system, the high attack rate in epidemic periods as well as the potentially severe complications [19]. Yearly influenza vaccination is recommended for all SLE patients; however, they may experience less protection. Adequate vaccine responses to influenza A and B, occurred in $39-41 \%$ of SLE patients compared to $71-94 \%$ controls, particularly in patients with higher ANA titers, IFN- $\alpha$ production, and number of ACR criteria for SLE $(>6)[20,21]$. Vaccination may be contraindicated during disease flare, as patients are less likely to develop a protective response [4]. While prevention of influenza infections may reduce the risk of development of pneumonia, there has been a concern that influenza vaccine may trigger SLE flares [22]. Several cases of SLE precipitation have been reported after immunization [23]. Nevertheless, most SLE patients with quiescent disease do not show an increase in both clinical and laboratory parameters after influenza vaccination as shown by the stability of SLE Disease Activity Index (SLEDAI) score [24,25].

\section{Pneumococcal vaccination}

Streptococcus pneumoniae infections are an important cause of morbidity and mortality worldwide particularly when subjects receiving aggressive long-term immunosuppressive therapy [26,27]. The 23-valent polysaccharide pneumococcal vaccine is recommended 
Page 2 of 4

in all SLE patients, as there is an increased risk of invasive pneumococcal infections, especially in patients with hyposplenia $[4,13]$.

It seems not to favor the clinical exacerbation of SLE [28]. The response rate of SLE patients to pneumococcal vaccination is only $47 \%$. Patients with high disease activity or using high dose corticosteroids were more likely to have inadequate vaccination responses. Poor responders can be vaccinated up to every 6 years as needed [4].

\section{Human Papilloma Virus (HPV) vaccination}

Given the increased risk of persistent HPV and developing abnormal cervical smears and squamous intraepithelial lesions of the cervix in SLE patient compared to healthy females, the HPV vaccine this vaccination should be encouraged $[14,29]$. It is a quadrivalent recombinant vaccine against HPV types 6, 11, 16 and 18.

It has been considered safe and well tolerated in a series of adolescents and young women with SLE, with no increase in mean SLEDAI scores [16,30]. Some cases of new-onset SLE in patients or disease flare have been reported two to four months following Gardasil vaccination. There are case reports of a temporal association of SLE onset or flare following Gardasil [31].

\section{Varicella Zoster Virus (VZV) vaccination}

Zostavax is a live-attenuated vaccine to prevent VZV in patients previously exposed to the virus [32,33]. The vaccine is thought to be safe, as it does not introduce new virus into a naive population, as seropositivity to VZV is confirmed prior to vaccination in immunosuppressed individuals [34]. Lupus patients with quiescent or low disease activity may benefit from Zostavax immunization, but caution should be taken by confirming prior infection with varicellaspecific IgG and delaying vaccination in patients with moderate to severe disease activity and/or on high doses of corticosteroids [34].

\section{Hepatitis B Virus (HBV) vaccination}

The HBV vaccine, non-live composite vaccine, has been studied in this population as well [14]. It may be administered according to the individual risk of acquiring this infection [13]. It is recommended in a three-dose series. SLE patients have reduced seroconversion, with adequate responses in $80 \%$ of children and $93 \%$ of adults, compared to $100 \%$ of controls $[35,36]$.

Some cases of new-onset SLE occurring within 2 months of HBV vaccination were reported [36]. In patients in whom rituximab treatment is planned, Ig levels should be tested before administration. If low baseline levels are detected, they should be monitored periodically, particularly in those at high risk, such as elderly patients or those undergoing concomitant glucocorticoid treatments [37].

\section{Others vaccines}

Other vaccinations required in SLE patients and all adults include tetanus toxoid in combination with diphtheria (Td) with a one-time acellular pertussis booster (Tdap) for all adults. SLE patients have reduced seroconversion rates ( $46 \%$ of recipients) and reduced antibody titers when compared to healthy controls [4].

\section{Chemoprophylaxis}

\section{Prophylaxis of tuberculosis (TB)}

Routine testing is recommended before glucocorticoids and immunosuppressive agents are used in patients coming from endemic areas [38]. Prophylaxis with isoniazid (INH) is controversial because the effectiveness of INH in preventing TB development is not well established in lupus patients, whilst INH has potential liver toxicity, especially if combined with other hepatotoxic drugs like azathioprine or methotrexate [39]. In addition, Bacillus Calmette-Guerin (BCG) vaccination has not proved effectiveness in preventing TB in adults and may be associated with adverse effects and therefore should be avoided in immunosuppressed patients [13]. However, Patients with prolonged glucocorticoid therapy and with a positive screen for latent TB should be treated with INH. Hepatic function must be closely monitored in these patients [40].

\section{Prevention of pneumocystis (PCP)}

PCP is greatly feared complication of immunosuppression [41]. Previous studies indicate that a dose of glucocorticoids $16 \mathrm{mg}$ increases the risk of PCP in non-HIV individuals and reported a PCP prevalence of $0.16 \%$ in SLE patients on cyclophosphamide [11,42]. Because of the risk of serious adverse events associated with antimicrobial prophylaxis for PCP, it is recommended that prophylaxis only be used when the risk of PCP in SLE patients with CD4+ Tcell count $<250 / \mu$ l, interstitial pulmonary fibrosis, high SLEDAI, severe nephritis, or with chronic use of prednisone $\geq 20 \mathrm{mg} /$ day $[43,44]$

\section{Antimalarial drugs}

Use of antimalarial drugs, including chloroquine and hydroxychloroquine, were shown to reduce incidence of infections in lupus [45]. SLE patients taking antimalarials are 16 times less likely to develop a major infection [46,47]. SLE patients who require more potent medications (corticosteroids or cytotoxic drugs) may still benefit from antimalarials, as they reduce infection rate, mortality, and have few side effects [4].

\section{Other preventive measures}

Antibiotic prophylaxis to prevent bacterial endocarditis is recommended prior to invasive dental procedures, as $1-4 \%$ of SLE patients develop endocarditis, a rate higher than patients with prosthetic heart valves [48]. Regardless of the cause of infections, adequate and prompt recognition and proper treatment of the infected patient are imperative [3]. Empiric therapy should be initiated for suspected bacterial infection, covering the most common and most deadly pathogens. Empiric antibiotic therapy in SLE patients with suspected bacteremia should include coverage of community-acquired causes (E. coli, $S$. aureus, Salmonella), as well as nosocomial causes (Pseudomonas, Klebsiella, Acinetobacter), as they are associated with reduced survival and poor long-term outcomes [49].

The use of glucocorticoids should be limited as much as possible, both in terms of daily dose and duration, with avoidance of long-term doses higher that $5 \mathrm{mg} /$ day, which are clearly related to many serious side effects, including infections [50]. Early identification of infections precipitating or coinciding with disease flare is essential, as a delay in antimicrobial therapy results in worse outcomes in SLE patients with infections. Procalcitonin (PCT) has a good negative predictive value 
Page 3 of 4

for bacterial infection in SLE patients with active disease, with a value $<0.17 \mathrm{ng} / \mathrm{ml}$ ruling out infection complicating SLE flare [4]. In SLE patients with quiescent disease, an increase in the CRP level is a preferred over PCT [7].

\section{Conclusion}

Infections represent the main cause of death in patients with SLE. Many infections of them can be prevented with timely immunization, reducing exposure to contagious contacts, screening for latent infection, and minimizing exposure to immunosuppressive drugs, which are essential to achieve optimal control of SLE activity. These measures will help to reduce the burden of major infections in SLE patients and consequently improve patient outcomes.

\section{Declaration of interest}

The authors report no conflicts of interest. The authors alone are responsible for the content and writing of the article.

\section{References}

1. Perl A (2010) Systems biology of lupus: mapping the impact of genomic and environmental factors on gene expression signatures, cellular signaling, metabolic pathways, hormonal and cytokine imbalance, and selecting targets for treatment. Autoimmunity 43: 32-47.

2. Conti F, Rezai S, Valesini G (2008) Vaccination and autoimmune rheumatic diseases. Autoimmun Rev 8: 124-128.

3. Murdaca G, Orsi A, Spanò F, Puppo F, Durando P, et al. (2014) Influenza and pneumococcal vaccinations of patients with systemic lupus erythematosus: Current views upon safety and immunogenicity. Autoimmun Rev 13: 75-84.

4. Caza T, Oaks Z, Perl A (2014) Interplay of infections, autoimmunity and immunosuppression in systemic lupus erythematosus. Int Rev Immunol 33: 330-363.

5. Khalifa M, Kaabia N, Bahri F, Ben JE, Bouajina E, et al. (2007) Infection in systemic lupus erythematosus. Med Mal Inf 37: 792-795.

6. Murdaca G, Colombo BM, Puppo F (2011) Emerging biological drugs: a new therapeutic approach for systemic lupus erythematosus. An update upon efficacy and adverse events. Autoimmun Rev 11: 56-60.

7. Zonana-Nacach A, Camargo-Coronel A, Yanez P, Sánchez L, JimenezBalderas FJ, et al. (2001) Infections in our patients with systemic lupus erythematosus: a prospective study. Lupus 10: 505-510.

8. Bernatsky S, Hudson M, Suissa S (2007) Anti-rheumatic drug use and risk of serious infections in rheumatoid arthritis. Rheumatology 46: 1157-1160.

9. Noël V, Lortholary O, Casassus P, Cohen P, Genereau T, et al. (2001) Risk factors and prognostic influence of infection in a single cohort of 87 adults with systemic lupus erythematosus. Ann Rheum Dis 60: 1141-1144.

10. Molina V, Shoenfeld Y (2005) Infection, vaccines and other environmental triggers of autoimmunity. Autoimmunity 38: 235-245.

11. Murray S, Schmajuk G, Trupin L, Gensler L, Katz P, et al. (2016) National lupus hospitalization trends reveal rising rates of herpes zoster and declines in pneumocystis pneumonia. PLoS One 5: 11.

12. van Assen S, Agmon-Levin N, Elkayam O, Cervera R, Doran MF, et al. (2011) EULAR recommendations for vaccination in adult patients with autoimmune inflammatory rheumatic diseases. Ann Rheum Dis 70: 414-422.

13. Danza A, Ruiz-Irastorza G (2013) Infection risk in systemic lupus erythematosus patients: susceptibility factors and preventive strategies. Lupus 22: 1286-1294.
14. Rotstein IH, Groot N, Lacerda MI, Wulffraat N, Pileggi G, et al. (2016) HPV infection and vaccination in Systemic Lupus Erythematosus patients: what we really should know. Pediatr Rheumatol Online J 14: 12.

15. Heijstek MW, Ott de Bruin LM, Bijl M, Borrow R, van der Klis F, et al. (2011) EULAR recommendations for vaccination in paediatric patients with rheumatic diseases. Ann Rheum Dis. 70: 1704-1712.

16. Soybilgic A, Onel KB, Utset T, Alexander K, Wagner-Weiner L (2013) Safety and immunogenicity of the quadrivalent HPV vaccine in female Systemic Lupus Erythematosus patients aged 12 to 26 years. Pediatr Rheumatol Online J 11: 29.

17. Abu-Shakra M (2009) Safety of vaccination of patients with systemic lupus erythematosus. Lupus 18: 1205-1208.

18. Duchini A, Goss JA, Karpen S, Pockros PJ (2003) Vaccinations for adult solid-organ transplant recipients: current recommendations and protocols. Clin Microbiol Rev 16: 357-364.

19. Wotton CJ, Goldacre MJ (2012) Risk of invasive pneumococcal disease in people admitted to hospital with selected immune-mediated diseases: record linkage cohort analyses. J Epidemiol Community Health 66: 1177-1181.

20. Holvast A, Huckriede A, Wilschut J, Horst G, De Vries JJ, et al. (2006) Safety and efficacy of influenza vaccination in systemic lupus erythematosus patients with quiescent disease. Ann Rheum Dis 65: 913-918.

21. Crowe SR, Merrill JT, Vista ES, Dedeke AB, Thompson DM, et al. (2011) Influenza vaccination responses in human systemic lupus erythematosus: impact of clinical and demographic features. Arthritis Rheum 63: 2396-2406.

22. Arom-Maor A, Shoenfeld Y (2001) Vaccination and systemic lupus erythematosus: the bidirectional dilemmas. Lupus 10: 237-240.

23. Perdan-Pirkmajer K, Thallinger GG, Snoj N, Cucnik S, Zigon P, et al. (2012) Autoimmune response following influenza vaccination in patients with autoimmune inflammatory rheumatic disease. Lupus 21: 175-183.

24. Holvast B, Huckriede A, Kallenberg CG, Bijl M (2007) Influenza vaccination in systemic lupus erythematosus: safe and protective? Autoimmun Rev 6: 300-305.

25. Del Porto F, Laganà B, Biselli R, Donatelli I, Campitelli L, et al. (2006) Influenza vaccine administration in patients with systemic lupus erythematosus and rheumatoid arthritis Safety and immunogenicity. Vaccine 24: 3217- 3223.

26. Vila-Córcoles A (2009) Vaccinate your child and save its grandparents from a heart attack? Current perspectives in anti-pneumococcal vaccination J Intern Med 266: 432-444.

27. Van Assen S, Elkayam O, Agmon-Levin N, Cervera R, Doran MF, et al. (2011) Vaccination in adult patients with auto-immune inflammatory rheumatic diseases: a systematic literature review for the European League Against Rheumatism evidence-based recommendations for vaccination in adult patients with autoimmune inflammatory rheumatic diseases. Autoimmun Rev 10: 341-352.

28. Elkayam O, Paran D, Caspi D, Litinsky I, Yaron M, et al. (2002) Immunogenicity and safety of pneumococcal vaccination in patientswith rheumatoid arthritis or systemic lupus erythematosus. Clin Infect Dis 34: 147-153.

29. Klumb EM, Pinto AC, Jesus GR, Araujo M Jr, Jascone L, et al. (2010) Are women with lupus at higher risk of HPV infection? Lupus 19: 1485-1491.

30. Rigante D, Esposito S (2015) Infections and systemic lupus erythematosus: binding or sparring partners? Int J Mol Sci 16: 17331-17343.

31. Soldevilla HF, Briones S, Navarra SV. Systemic lupus erythematosus following HPV immunization or infection? Lupus 21: 158-161.

32. Chakravarty EF, Michaud K, Katz R, Wolfe F (2013) Increased incidence of herpes zoster among patients with systemic lupus erythematosus. Lupus 22: 238-244.

33. Oxman MN, Levin MJ, Johnson GR, Schmader KE, Straus SE, et al. (2005) A vaccine to prevent herpes zoster and postherpetic neuralgia in older adults. N Engl JMed 352: 2271-2284. 
Citation: EL Fane M, Sodqi M, Chakib A, EL Filali KM (2017) Prevention of Infection in Lupus Patients. J Antimicrob Agents 3: 131. doi: $10.4172 / 2472-1212.1000131$

Page 4 of 4

34. Cogman AR, Chakravarty EF (2013) The case for Zostavax vaccination in systemic lupus erythematosus. Vaccine 31: 3640-3643.

35. Aytac MB, Kasapcopur O, Aslan M, Erener-Ercan T, Cullu-Cokugras F, et al. (2011) Hepatitis B vaccination in juvenile systemic lupus erythematosus. Clin Exp Rheumatol 29: 882-886.

36. Kuruma KA, Borba EF, Lopes MH, de Carvalho JF, Bonfá E (2007) Safety and efficacy of hepatitis B vaccine in systemic lupus erythematosus. Lupus 16: 350-354.

37. Buch MH, Smolen JS, Betteridge N, Breedveld FC, Burmester G, et al. (2011) Updated consensus statement on the use of rituximab in patients with rheumatoid arthritis. Ann Rheum Dis 70: 909-920.

38. Mosca M, Tani C, Aringer M, Bombardieri S, Boumpas D, et al. (2010) European League Against Rheumatism recommendations for monitoring patients with systemic lupus erythematosus in clinical practice and in observational studies. Ann Rheum Dis 69: 1269-1274.

39. Mok MY, Lo Y, Chan TM, Wong WS, Lau CS (2005) Tuberculosis in systemic lupus erythematosus in an endemic area and the role of isoniazid prophylaxis during corticosteroid therapy. J Rheumatol 32: 609-615.

40. Barber C, Gold WL, Fortin PR (2011) Infections in the lupus patient: Perspectives on prevention. Curr Op Rheumatol 23: 358-365.

41. Mansharamani NG, Garland R, Delaney D, Koziel H (2000) Management and outcome patterns for adult pneumocystis carinii pneumonia, 1985 to 1995: Comparison of HIV-associated cases to other immunocompromised states. Chest 118: 704-711.

42. Gupta D, Zachariah A, Roppelt H, Patel AM, Gruber BL (2008) Prophylactic antibiotic usage for pneumocystis jirovecii pneumonia in patients with systemic lupus erythematosus on cyclophosphamide: a survey of us rheumatologists and the review of literature. J Clin Rheumatol 14: 267-272.

43. Lertnawapan R, Totemchokchyakarn K, Nantiruj K, Janwityanujit S (2009) Risk factors of Pneumocystis jiroveci pneumonia in patients with systemic lupus erythematosus. Rheumatol Int 29: 491-496.

44. Green H, Paul M, Vidal L, Leibovici L (2007) Prophylaxis of pneumocystis pneumonia in immunocompromised non-hiv-infected patients: systematic review and meta-analysis of randomized controlled trials. Mayo Clin Proc 82: 1052-1059.

45. Ruiz-Irastorza G, Ramos-Casals M, Brito-Zeron P, Khamashta MA (2010) Clinical efficacy and side effects of antimalarials in systemic lupus erythematosus: a systematic review. Ann Rheum Dis 69: 20-28.

46. Ruiz-Irastorza G, Olivares N, Ruiz-Arruza I, Martinez-Berriotxoa A, Egurbide MV, et al. (2009) Predictors of major infections in systemic lupus erythematosus. Arthritis Res Ther: 11: 109.

47. Rolain J, Colson P, Raoult D (2007) Recycling of chloroquine and its hydroxyl analogue to face bacterial, fungal and viral infections in the 21st century. Int J Antimicrob Agents 30: 297-308.

48. Zysset MK, Montgomery MT, Redding SW, Dell'Italia LJ (1987) Systemic lupus erythematosus: a consideration for antimicrobial prophylaxis. Oral Surg Oral Med Oral Pathol 64: 30-34.

49. Chen MJ, Tseng HM, Huang YL, Hsu WN, Yeh KW, et al. (2008) Longterm outcome and short-term survival of patients with systemic lupus erythematosus after bacteraemia episodes: 6-yr follow-up. Rheumatology 47: 1352-1357.

50. Ruiz-Irastorza G, Danza A, Khamashta M (2012) Glucocorticoid use and abuse in SLE. Rheumatology 51: 1145-1153. 